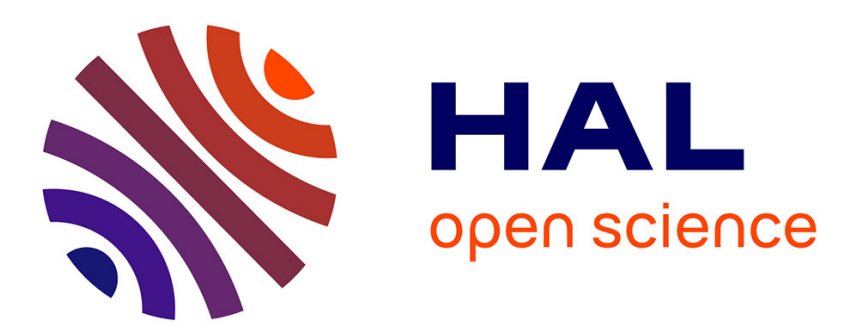

\title{
Le Bois dans le contexte des matériaux de construction. Conférence présentée à l'ENGREF le 8 octobre 1999...
}

\author{
Pascal Triboulot, Benoît Reitz
}

\section{To cite this version:}

Pascal Triboulot, Benoît Reitz. Le Bois dans le contexte des matériaux de construction. Conférence présentée à l'ENGREF le 8 octobre 1999.... Revue forestière française, 2000, 52 (4), pp.291-302. $10.4267 / 2042 / 5363$. hal-03443447

\section{HAL Id: hal-03443447 \\ https://hal.science/hal-03443447}

Submitted on 23 Nov 2021

HAL is a multi-disciplinary open access archive for the deposit and dissemination of scientific research documents, whether they are published or not. The documents may come from teaching and research institutions in France or abroad, or from public or private research centers.
L'archive ouverte pluridisciplinaire HAL, est destinée au dépôt et à la diffusion de documents scientifiques de niveau recherche, publiés ou non, émanant des établissements d'enseignement et de recherche français ou étrangers, des laboratoires publics ou privés. 


\title{
LE BOIS DANS LE CONTEXTE DES MATÉRIAUX DE CONSTRUCTION
}

\author{
P. TRIBOULOT - B. REITZ
}

\section{NDLR}

Ce texte correspond à une présentation qui a été faite le 8 octobre 1999 dans les locaux de l'École nationale du Génie rural, des Eaux et des Forêts (ENGREF) de Nancy et dans le cadre de la manifestation "Le bois : essence de l'Art" organisée par l'Association pour la Recherche sur le Bois en Lorraine (ARBOLOR) à l'occasion de l'année de l'École de Nancy (au sens de l'école d'Art nouveau).

\section{LE BOIS : MATÉRIAU DE TOUJOURS}

Plus que tout autre, le matériau bois évoque le temps et l'âge : le temps parce que sa production est assurée par la croissance des arbres qui deviennent récoltables après une période assez longue, variable selon l'espèce et la station ; l'âge, parce qu'il est, dans l'histoire, celui qui est utilisé par l'homme depuis le plus longtemps. C'est sans doute parce que la dépense d'énergie nécessaire à sa transformation est particulièrement faible (il suffit de rompre les branches, de couper, de scier...), par comparaison avec l'élaboration des métaux ou l'exploitation des minéraux, qu'il a été mis en œuvre très tôt par l'homme, que ce soit pour se défendre, pour chasser ou s'abriter. C'est aussi parce que, dès l'origine des temps, la forêt a joué son rôle de "forêt-abri" et de "forêt nourricière" que religieux, brigands, marauds, contestataires de tout poil, et bien avant encore, l'ancêtre de l'honnête homme y ont trouvé refuge et matière première en abondance.

C'est donc l'histoire d'une rencontre entre l'homme et la forêt qui initia, depuis l'aube de l'humanité, une "filière-bois" qui démarra exactement le jour où une première lueur d'intelligence brilla dans la pupille caverneuse de nos ancêtres descendus des arbres. Cette filière s'organisa immédiatement autour de trois axes principaux :

- filière biens d'équipement et construction (armes, outils, huttes...),

- filière agro-alimentaire (cueillette, baies, champignons...),

- filière-bois-énergie (le feu...).

À l'aube du troisième millénaire (Sandoz, 1994), un défi extrême s'impose à l'humanité : nourrir, protéger plus de 10 milliards d'individus. Le relever passe par l'adéquation permanente entre agriculture, matières premières, industrie et environnement. Dans ce contexte, la forêt, élément régulateur de l'environnement et fournisseur de matière première et d'énergie, jouera un rôle majeur, avec 


\section{P. TRIBOULOT - B. REITZ}

vraisemblablement une inversion des flux (dont le bois) entre l'hémisphère Nord et l'hémisphère Sud. Ce défi devrait donc s'appuyer sur une filière-bois forte, redéveloppée selon trois axes :

- bois-construction,

- bois-chimie,

- bois-énergie.

On conviendra qu'en plusieurs dizaines de milliers d'années, cette "filière-bois" présente une constance assez exceptionnelle et sans doute unique, dans ses stratégies, son organisation et ses objectifs !

Nombreux sont les exemples qui permettent de traduire cette constance ; la fabrication d'outils ou de manches d'outils est sans doute l'usage technologique le plus ancien des matériaux tirés de la forêt : une souche d'arbre est devenue massue, une branche brisée par le vent élément de défense (Noël et Bocquet, 1987). Des traces les plus anciennes de manches d'outils datant du Mésolithique à aujourd'hui, rien n'a mieux permis que le bois de garantir, à la fois, des caractéristiques de flexion dynamique et une résilience élevée. Aucun matériau ne permet aussi bien d'absorber dans cet usage les chocs, de supprimer les vibrations et d'assurer un confort et une ergonomie adaptée à l'utilisateur (Facom, 1994). On met ici l'accent sur la complexité qui s'affirme aujourd'hui en matière de choix des matériaux pour un usage donné. La science des matériaux ne travaille pas sur un seul axe (par exemple la recherche d'une propriété mécanique élevée) ; elle cherche au contraire et en permanence un compromis optimisé entre plusieurs propriétés souvent contradictoires (un matériau à la dureté élevée a par exemple un comportement fragile ; un composite artificiel a un module d'élasticité très élevé, mais un coût proportionnel, etc.).

L'usage du bois a été longtemps lié à l'arbre. L'homme a observé et compris tout le parti qu'il pouvait tirer de cette architecture particulière et exceptionnelle. La nature a pourvu l'arbre d'un fût rond. À cet égard, l'inventeur de la précontrainte ne fut sans doute pas Eugène Freyssinet (Picon, 1997), mais bien l'arbre qui, à travers son organisation structurelle, apporte un éclairage intéressant sur l'équilibre des contraintes sans lequel il ne pourrait s'élever. Parfois l'homme força la nature pour mieux en tirer profit, avec à l'origine une complicité certaine : la disposition naturelle des branches, des racines était utilisée pour concevoir immédiatement un siège, un élément de charpente, l'étambot d'un vaisseau, une fourche à fourrage, etc.

Puis l'homme et son insatisfaction permanente, source de créativité, a inventé le bois... rectangulaire ; l'industrie du sciage était née. Cette dernière n'a cessé de se développer et de s'adapter aux produits générés par la forêt d'une part, aux attentes des prescripteurs d'autre part. Les besoins en

Tableau I

Caractéristiques d'isolation thermique des matériaux

\begin{tabular}{|c|c|}
\hline Matériau & $\lambda: \mathrm{W} /\left(\mathrm{m} .{ }^{\circ} \mathrm{C}\right)$ \\
\hline Acier $\ldots \ldots \ldots \ldots$ & 52 \\
\hline Aluminium $\ldots \ldots$ & 230 \\
\hline Granit $\ldots \ldots \ldots$ & 3,50 \\
\hline Béton $\ldots \ldots \ldots$ & 1,75 \\
\hline Plâtre $\ldots \ldots \ldots$ & 0,50 \\
\hline Chêne $\ldots \ldots \ldots$ & 0,23 \\
\hline Épicéa $\ldots \ldots \ldots$ & 0,14 \\
\hline Balsa $\ldots \ldots \ldots$ & 0,054 \\
\hline Laine de roche $\ldots$ & 0,05 \\
\hline
\end{tabular}

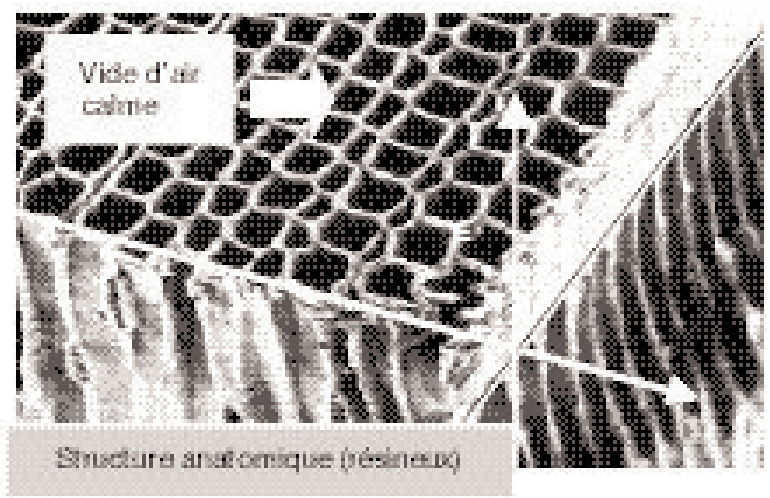


sections, longueurs, surfaces n'ont cessé de croître et ont conduit aux techniques de reconstitution bien maîtrisées aujourd'hui : lamellé-collé, contreplaqués, parallam, etc.

Si l'âge du bronze, l'âge du fer sont à ce jour dépassés, l'âge du bois est une telle évidence d'actualité que les experts s'efforcent de l'ignorer. Depuis l'origine, les arbres et le bois représentent l'un des succès majeurs de l'évolution des espèces (Gordon, 1994). Ils nous sont devenus si familiers que nous ne réalisons plus à quel point il y a là un matériau ultra perfectionné, à la structure ingénieuse et optimisée : un matériau composite naturel et renouvelable, vraie réponse à l'ensemble des problèmes des sociétés et civilisations qui nous ont précédés et vraie réponse aux problématiques du siècle qui s'avance. Matériau de toujours est, dans ce contexte, la qualification la plus pertinente que l'on puisse donner au bois.

\section{LE BOIS MATÉRIAU COMPOSITE NATUREL}

C'est l'anatomie du bois qui en fait un véritable matériau "high-tech", matériau composite naturel dont le modèle d'optimisation sert de base à de nombreux matériaux composites artificiels.

Ces matériaux composites naturels ou artificiels présentent en effet les points communs suivants :

- structure au caractère périodique marqué,

- fibres aux propriétés mécaniques élevées, englobées dans une matrice en général thermodurcissable. (Ce sera l'ensemble cellulose/hémi-cellulose/lignine dans le composite bois massif).

Sur cette base de définition, à un premier niveau d'observation, la section droite du tronc de l'arbre met en évidence les accroissements annuels dont la largeur et l'hétérogénéité relèvent du caractère saisonnier des périodes de croissance et des aléas climatiques. La structure alvéolaire périodique du bois, dans la section droite, apparaît à des niveaux de grossissement faibles ; on est alors en présence d'un matériau constitué d'éléments tubulaires orientés dans la direction longitudinale (direction de croissance en hauteur de l'arbre). Ces éléments sont renforcés par d'autres constituants anatomiques, les rayons ligneux, orientés dans la direction radiale. La paroi des éléments du bois est constituée de différentes couches, chaque couche étant caractérisée par un angle d'enroulement de "micro-fibrilles de cellulose".

C'est sur ce modèle d'enroulement en couches que repose aujourd'hui la technique de base pour la fabrication des éléments tubulaires réalisés en composites artificiels (mâts de bateau en fibres de kevlar par exemple).

De manière générale, toutes les performances physico-mécaniques du bois, pertinentes pour la construction, découlent de cette anatomie sophistiquée.

La structure anatomique du matériau explique par exemple et à elle seule les propriétés d'isolant qu'offre le bois. Les vides d'air liés à la porosité du bois permettent d'obtenir des coefficients de conductibilité thermiques exceptionnels en comparaison de ceux des matériaux structurels concurrents. Le tableau I (p. 292) rassemble les valeurs du coefficient $\lambda$ exprimé en watts par mètre et par degré Celcius, $\mathrm{W} /\left(\mathrm{m} .{ }^{\circ} \mathrm{C}\right)$ qui donne la quantité de chaleur pouvant traverser en une heure un mètre d'épaisseur d'un matériau sur une surface de $1 \mathrm{~m}^{2}$ lorsque la différence de température entre les deux faces est de $1{ }^{\circ} \mathrm{C}$ (un $\lambda$ fort caractérise un matériau très mauvais isolant thermique) (Triboulot, 2000). 


\section{LE BOIS MATÉRIAU PÉRENNE}

Dans des conditions optimales respectant les principes de base de la conception des ouvrages, le bois peut durer des siècles sans détérioration notable ; I'histoire du patrimoine bâti est là aussi explicite. L'une des plus anciennes constructions en bois, symbole de durabilité, est le sanctuaire bouddhiste Horyu-ji près de Nara au Japon. Témoin de l'architecture du VII ${ }^{\mathrm{e}}$ siècle, il va sans encombre vers le III ${ }^{\mathrm{e}}$ millénaire (Natterer et al., 1995).

Les facteurs d'agression du bois sont bien connus : l'eau et le soleil avec, en corollaire, les champignons et les insectes. Dans le même esprit, les autres matériaux de construction ont aussi à lutter contre les nuisances : l'oxydation, la corrosion, l'érosion, la photodégradation. Pour faire face, les secteurs correspondants développent eux aussi des techniques d'entretien, préventives ou curatives, et des modalités de préservation : il faut 50 tonnes de peinture tous les 7 ans pour assurer l'entretien de la célèbre Tour Eiffel. Dans le cas des structures en béton, le phénomène de corrosion des armatures, consécutif à la carbonatation naturelle des bétons, pose d'énormes problèmes d'entretien : il fait gonfler les armatures et éclater le béton. La majorité des ouvrages d'art antérieurs aux années 1980 sont confrontés aujourd'hui à ce problème majeur.

Pour le bois, les attaques biologiques sont de deux types:

- les attaques cryptogamiques : elles se développent au-delà d'un seuil d'humidité minimal dans le bois (entre 20 et $30 \%$, selon les conditions de température et, parfois, d'éclairement),

- les attaques d'insectes : elles sont favorisées par des températures suffisamment élevées pour permettre la reproduction et le développement des larves.

Tableau II

Durabilité naturelle de quelques essences

\begin{tabular}{|c|c|c|c|c|}
\hline \multicolumn{5}{|c|}{ Absence de traitement, quelle essence pour quel emploi ? } \\
\hline & "Classe 1" & "Classe 2" & "Classe 3" & "Classe 4" \\
\hline $\begin{array}{l}\text { Domaines possibles } \\
\text { d'utilisation } \\
\text { définissant la classe } \ldots . .\end{array}$ & $\begin{array}{l}\text { Menuiseries } \\
\text { intérieures } \\
\text { Parquets } \\
\text { Lambris }\end{array}$ & $\begin{array}{l}\text { Charpentes } \\
\text { Bardage } \\
\text { vertical }\end{array}$ & $\begin{array}{l}\text { Bardage } \\
\text { Menuiseries } \\
\text { extérieures }\end{array}$ & $\begin{array}{l}\text { Pieds de poteaux } \\
\text { Caillebotis } \\
\text { Mobiliers } \\
\text { urbains }\end{array}$ \\
\hline \multicolumn{5}{|c|}{ Résineux } \\
\hline \multicolumn{5}{|l|}{ Douglas ............ } \\
\hline \multicolumn{5}{|l|}{ Mélèze $\ldots \ldots \ldots \ldots \ldots$} \\
\hline \multicolumn{5}{|l|}{$\begin{array}{l}\text { Western Red Cedar .... } \\
\text { (Thuya plicata) }\end{array}$} \\
\hline \multicolumn{5}{|l|}{ Sapin ............... } \\
\hline \multicolumn{5}{|c|}{ Feuillus } \\
\hline \multicolumn{5}{|l|}{ Châtaignier ......... } \\
\hline \multicolumn{5}{|l|}{ Chêne $\ldots \ldots \ldots \ldots \ldots$} \\
\hline \multicolumn{5}{|l|}{ Robinier (faux acacia) } \\
\hline \multicolumn{5}{|l|}{ Doussié ............. } \\
\hline Iroko ............. & & & & \\
\hline
\end{tabular}

Classes de risque couvertes par l'essence, utilisation possible sans traitement. Document non contractuel, se référer à la norme NF EN 350, 351 et 335. 
D'une manière générale, les dispositions constructives permettent d'éviter le dépassement du seuil d'humidité à partir duquel une attaque cryptogamique est possible ; elles reposent sur des règles simples :

- la limitation des reprises d'humidité (par le sol et par exposition directe),

- la ventilation des bois et l'évacuation rapide de l'eau lorsque le bois est exposé,

- l'utilisation de bois possédant une durabilité naturelle suffisante ou traité de manière adéquate.

Pour une grande partie des usages actuels, il est possible de faire appel à des essences ayant naturellement le niveau de durabilité requis pour répondre aux exigences de la classe de risque et compatible avec la durée de vie souhaitée en l'absence de tout traitement. Le tableau II (p. 294) dresse l'inventaire uniquement sur quelques essences courantes des possibilités offertes aux concepteurs (CTBA, 1998).

Si les forêts nationales sont capables d'abriter quelques essences naturellement durables, force est de constater que l'offre et la disponibilité des plus intéressantes d'entre elles (Mélèze, Robinier) restent trop limitées. II y a là matière à réflexion en matière d'aménagement forestier.

\section{LE BOIS MATÉRIAU SÛR}

Le bois est certes un bon combustible, mais il possède dans ses usages en structures l'avantage unique vis-à-vis de tous les autres matériaux de construction de conserver ses propriétés portantes à haute température pendant l'incendie ; sa réaction est alors prévisible et planifiable. Les éléments porteurs brûlent en périphérie (de l'ordre de $0,5 \mathrm{~mm} / \mathrm{mn}$ ), ne s'effondrent pas, permettent l'évacuation des occupants (contrairement à l'acier qui voit ses capacités portantes décliner immédiatement avec l'augmentation de la température, l'incendie du CES Pailleron en étant la tragique illustration). Le bois ne dégage pas de gaz toxiques contrairement au PVC, ne se dilate pas contrairement à l'acier (qui provoque aux interfaces avec le béton des éclatements). Dans les constructions bois conséquentes, les assemblages métalliques sont maintenant protégés par l'intégration complète de l'assemblage en métal dans le bois ou par bouchonnages en bois des tiges de connexion. C'est donc le bois qui protège l'acier vis-à-vis de l'incendie et pas l'inverse. Pour les petites sections de bois plus vulnérables, les règles de conception (plâtre, vernis ignifugés...) permettent d'apporter une réponse technique.

\section{LE BOIS MATÉRIAU PERFORMANT}

Comparer les propriétés mécaniques du bois, des matériaux de construction et des composites artificiels, sur la base simpliste du module d'élasticité ou de la contrainte de rupture par exemple, aboutit à des conclusions sans appel mais dénuées de sens. Le tableau III (p. 296) présente cette comparaison sur les trois matériaux de base de la construction. Sur ces critères classiques et en dehors de tout contexte, le bois présente des niveaux de performance médiocres.

Un acier de construction à $210000 \mathrm{MPa}$ (mégapascal) de module d'élasticité, et un résineux à moins de 10000 MPa sont-ils raisonnablement comparables (Triboulot et Duchanois, 1996) ? Cette comparaison est seulement réaliste à partir du contexte d'utilisation et par rapport :

- aux caractéristiques spécifiques (module et contrainte ramenés à la densité),

- à l'usage ou à la fonction,

- au meilleur compromis "performances mécaniques/coûts",

- au bilan énergétique (et écologique). 


\begin{tabular}{|c|c|c|c|}
\hline Matériau & Acier & Béton & Bois \\
\hline & S355 & B30 & $\begin{array}{c}\text { Lamellé-collé } \\
\text { GL28 }\end{array}$ \\
\hline \multicolumn{4}{|l|}{ Propriétés de résistance (MPa) } \\
\hline Flexion $\ldots \ldots \ldots \ldots \ldots \ldots$ & 270 & 18 & 12,3 \\
\hline Traction axiale $\ldots \ldots \ldots \ldots \ldots$ & 270 & 2,4 & 8,6 \\
\hline Traction perpendiculaire $\ldots . .$. & 270 & 2,4 & 0,2 \\
\hline Compression axiale $\ldots \ldots \ldots \ldots$ & 270 & 18 & 12,3 \\
\hline Compression perpendiculaire ... & 270 & 18 & 2,4 \\
\hline Cisaillement $\ldots \ldots \ldots \ldots \ldots \ldots$ & 213 & 1,8 & 1,3 \\
\hline \multicolumn{4}{|l|}{ Propriétés de rigidité (MPa) } \\
\hline Module axial moyen $\ldots \ldots \ldots$ & 210000 & 11500 & 12600 \\
\hline Module transversal moyen $\ldots$. & 210000 & 11500 & 1020 \\
\hline Module de cisaillement moyen . . & 8100 & 4800 & 790 \\
\hline
\end{tabular}

À titre d'exemple, nous traiterons ici des poteaux porteurs, configuration courante de mise en œuvre des matériaux dans la construction. Le concepteur s'attache à rechercher le matériau le plus léger possible (facilité de mise en œuvre), résistant en compression et ne présentant pas de risque de flambement.

On démontre simplement qu'un critère de qualité pour un matériau mis en œuvre sous forme de poteau, colonne ou pilier s'exprime en rapportant, pour ce matériau, la racine carrée du module d'élasticité à la masse volumique.

La charge limite F, susceptible de provoquer le flambement, est donnée par l'expression d'Euler :

$$
F=k \pi^{2} \quad \frac{E l}{L^{2}}
$$

où $\mathrm{k}$ est une constante qui dépend des conditions de liaison du poteau au sol, $\mathrm{E}$ est le module d'élasticité du matériau, I l'inertie de la section droite, L la hauteur du poteau chargé. Pour une géométrie donnée de la section droite du poteau et une masse volumique "ro (en symbole)" du matériau, la masse du poteau prend la forme :

$$
m=k^{\prime} \frac{\rho}{\sqrt{E}} L^{2} \sqrt{F}
$$

Cette expression revient alors à rechercher pour m minimum, un ratio en $(\rho / \sqrt{E})$ qui soit minimum ou un ratio inverse en $(\sqrt{E} / \rho)$ qui soit maximum.

Le tableau IV (p. 297) donne les valeurs obtenues pour quelques matériaux de construction et concernant ce critère de qualité dans cette application (Gordon, 1994).

Dans d'autres configurations du type "panneaux", le flambement ne peut se produire que perpendiculairement au plan du panneau; une même démarche conduit à la recherche d'un critère de qualité qui s'exprime dans ce contexte en racine cubique de E/densité. Dans ces deux configurations, les résultats obtenus avec les résineux sont nettement supérieurs à ce qui se fait de plus 
classique, l'acier, le béton, l'aluminium, mais également aux matériaux composites artificiels (fibres de verres, fibres de Kevlar...) considérés comme plus modernes. Ces chiffres traduisent le niveau de performance extraordinaire du matériau bois ; malheureusement la religion du progrès, qui veut que ce soient les matériaux les plus nouveaux, les plus artificiels, qui soient forcément les meilleurs, a occulté cette réalité mécanique.

Par ailleurs, on comprend à travers ces chiffres pourquoi le bois issu des arbres les plus hauts du monde (à l'exemple des Séquoias de Californie) est en général à densité faible.

Une autre réflexion porte sur la notion de "qualité des bois" ; la densité seule a été trop souvent dans le passé l'indicateur de cette qualité. Dans la majorité des usages constructifs, ce seul critère est insuffisant ; ce sont les différentes formes des ratio propriétés/densité, liées aux configurations de mise en œuvre, qui doivent être prises en compte.

\section{LE BOIS MATÉRIAU “ÉCO-LOGIQUE”}

L'importance des écobilans dans la conception de produits et dans la démarche constructive à l'image du label HQE (Haute Qualité Environnementale) devrait ouvrir de nouvelles perspectives pour le bois et le positionner de manière objective dans l'ensemble des matériaux de construction. Si un écobilan se définit comme "le bilan quantifié des flux matière et énergie aux frontières d'un système, d'un produit ou d'un procédé ", ou, plus prosaïquement, du berceau à la tombe, le bois a un rôle essentiel à jouer. Les premières études montrent que le bois présente, par rapport à l'acier, l'aluminium, les matériaux issus de la pétrochimie, un "écobilan" favorable (Guinard, 1995). Les seules restrictions portent sur les générations anciennes d'adjuvants, colles, peintures, produits de préservation utilisés dans la mise en œuvre du matériau. Les chiffres et arguments ne manquent pas ; le tableau V (p. 298) compare l'énergie nécessaire pour produire une tonne de matière (Legovic, 1995) ; ces chiffres parlent d'eux-mêmes.

Dans la construction, le label "HQE" implique une vision prospective. Construire sans songer à économiser les ressources non renouvelables, en amont, ou sans connaître la destination des matériaux, en fin de vie, n'est plus envisageable. Au-delà des aspects techniques, nous sommes là face à une véritable révolution culturelle. Des matériaux utilisés couramment dans le bâtiment comme le zinc ou le cuivre présentent des potentiels de disponibilité de l'ordre de quelques dizaines d'années seulement au rythme actuel de leur exploitation.

Tableau IV

Critères de performance des matériaux

\begin{tabular}{|c|c|c|c|c|}
\hline Matériaux & $\begin{array}{l}\text { Module } \\
\text { d'élasticité } \\
\text { E (MPa) }\end{array}$ & $\begin{array}{c}\text { Densité } \\
(\rho)\end{array}$ & $\begin{array}{c}\text { Critère de qualité } \\
\text { pour une colonne } \\
\text { Racine carrée } \\
\text { de E/p }\end{array}$ & $\begin{array}{c}\text { Critère de qualité } \\
\text { pour un panneau } \\
\text { Racine cubique } \\
\text { de } E / \rho\end{array}$ \\
\hline Acier $\ldots \ldots \ldots \ldots \ldots$ & 210000 & 7,8 & 59 & 7,7 \\
\hline Titane.......... & 120000 & 4,5 & 77 & 11,0 \\
\hline Aluminium ........... & 73000 & 2,8 & 99 & 15,0 \\
\hline Béton $\ldots \ldots$ & 15000 & 2,5 & 49 & 10,0 \\
\hline $\begin{array}{l}\text { Matériau composite } \\
\text { artificiel ............ }\end{array}$ & 200000 & 2,0 & 225 & 10,0 \\
\hline Résineux léger $\ldots \ldots \ldots$ & 10000 & 0,35 & 290 & 61,4 \\
\hline Feuillus $\ldots \ldots \ldots \ldots$ & 12000 & 0,65 & 170 & 35,5 \\
\hline
\end{tabular}




\begin{tabular}{|c|c|}
\hline Matériau & $\begin{array}{c}\text { Énergie pour produire } \\
\text { une tonne du matériau (mégajoules) }\end{array}$ \\
\hline Résineux indigène $\ldots \ldots \ldots \ldots \ldots \ldots$ & 1 \\
\hline Béton $\ldots \ldots \ldots \ldots \ldots \ldots \ldots \ldots$ & 4 \\
\hline Polyéthylène . . . . . . . . . . . . . . . . & 45 \\
\hline Acier $\ldots \ldots \ldots \ldots \ldots \ldots \ldots \ldots$ & 60 \\
\hline Aluminium $\ldots \ldots \ldots \ldots \ldots \ldots$ & 250 \\
\hline Fibres de carbone $\ldots . . . .$. & 4000 \\
\hline
\end{tabular}

Le caractère renouvelable de la matière ligneuse positionne le bois de manière forte dans cette logique environnementale. Bien entendu les conséquences de la dernière tempête de décembre 1999 marquent les esprits. II est important cependant de relativiser les effets dévastateurs de ces dérèglements climatiques. Le tableau VI (ci-dessous) précise les données relatives à la disponibilité du matériau en France (Bianco, 1998) et chiffre ce caractère indéfiniment renouvelable du bois, et ce, malgré les aléas de l'actualité récente.

Tableau VI

Production de matière ligneuse en France

\begin{tabular}{|c|c|}
\hline Volume sur pied (avant décembre 1999) . . . . . . . . & 2000 millions de $\mathrm{m}^{3}$ \\
\hline $\begin{array}{l}\text { Accroissement biologique annuel } \ldots \ldots \ldots \ldots \ldots \\
\text { Production de "I'usine à bois" française } \ldots \ldots \ldots \ldots\end{array}$ & $\begin{array}{l}85 \text { millions de } \mathrm{m}^{3} \\
232000 \mathrm{~m}^{3} / \text { jour }\left(^{*}\right)\end{array}$ \\
\hline Récolte annuelle $\ldots \ldots \ldots \ldots \ldots \ldots \ldots$ & 47 millions de $\mathrm{m}^{3}$ \\
\hline Consommation intérieure française $\ldots \ldots \ldots \ldots \ldots$ & 55 millions de $\mathrm{m}^{3}$ \\
\hline Tempête de décembre 1999 & $\begin{array}{l}\text { Soit: } \\
7 \% \text { du volume sur pied } \\
19 \text { mois d'accroissement biologique }\end{array}$ \\
\hline
\end{tabular}

$\left.{ }^{*}\right)=75 \%$ du volume annuel scié par la plus grosse scierie française.

La contribution de la forêt et du bois à la régulation de l'effet de serre n'est plus à démontrer. Si le dioxyde de carbone représente à lui seul près de $60 \%$ des gaz à effet de serre, les conséquences liées à des choix de matériaux dans la construction prennent rapidement de l'importance, les dysfonctionnements climatiques récents en sont l'illustration.

L'ensemble des figures 1 à 4 (p. 299) présente les évolutions mesurées de la température moyenne et des concentrations de $\mathrm{CO}_{2}$ atmosphérique, les climatologues s'accordent aujourd'hui sur la quasi parfaite corrélation entre les deux phénomènes; les mesures effectuées sur les derniers millénaires confortent cette analyse. La persistance et l'évolution de l'effet de serre conduisent également à s'interroger sur les dérèglements climatiques qui en résultent.

En parallèle de ces deux courbes sont présentées :

- l'évolution des coûts des dommages liés aux catastrophes naturelles sur la période 19801998 (Hileman, 1999), 
- l'évolution des conséquences forestières des grandes tempêtes sur le siècle (aspect historique des chablis, http://www.onf.fr).

Sur la figure 4 (où les segments de droite n'ont pas de signification et qui se veut uniquement un indicateur de tendance), la fréquence des événements commence à augmenter vers les années 1960, avec un cumul au-delà des années 1970 pour atteindre les sommets de décembre 1999. II y a là matière à réflexion sur la probabilité forte d'être à nouveau confronté à des dysfonctionnements climatiques majeurs dans les prochaines décennies (Desbois et Pouradier, 2000).

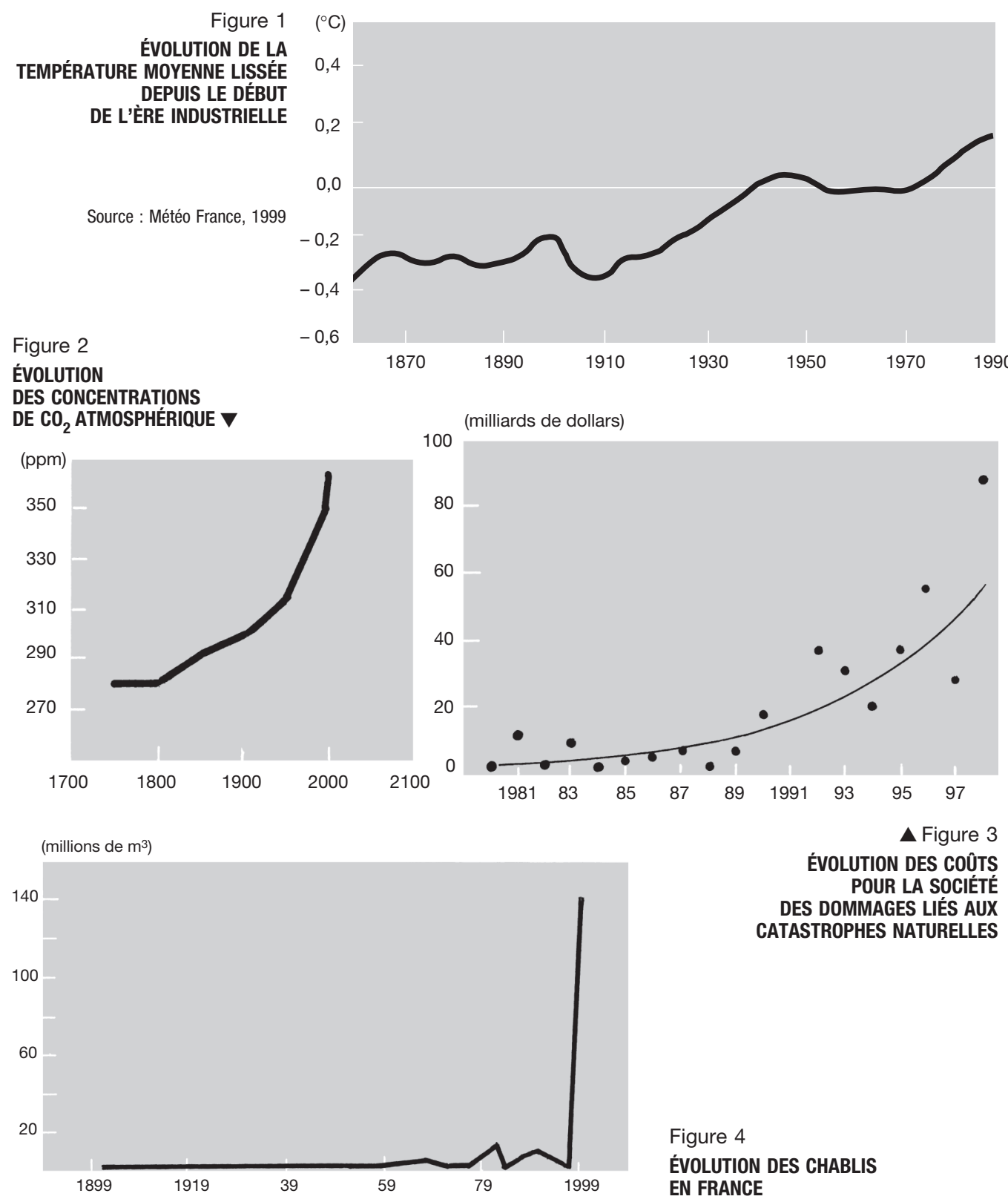


Les coûts induits par les catastrophes naturelles présentent une évolution parallèle et exponentielle ; ce coût deviendra à terme insupportable pour la Société, il sera urgent d'envisager (s'il n'est pas trop tard) une autre stratégie de choix en matière d'utilisation de matériaux (fossiles ou renouvelables) dans les secteurs où les gros volumes sont utilisés (c'est le cas de la construction). Dans ce contexte, le bois présente là encore des perspectives de développement considérables.

On ne reviendra pas ici sur le bilan dans le cycle du carbone, globalement nul pour ce qui concerne l'utilisation du bois-énergie ou résultant du cycle photosynthèse - décomposition biologique du bois des arbres en fin de vie. Les possibilités de créer de véritables "puits de carbone" sont réelles à partir du développement systématique du bois dans la construction. En France, la "loi sur l'air" est dans ce sens une première avancée significative, mais mériterait, compte tenu des enjeux, des décrets d'application très dynamiques.

Le tableau VII (ci-dessous) précise les effets sur l'atmosphère (donc sur les dysfonctionnements climatiques) des choix qui peuvent être faits en termes de matériaux de structure (Triboulot, 2000).

Tableau VII

Effet environnemental des matériaux de construction

\begin{tabular}{|c|c|c|c|}
\hline \multirow{2}{*}{ Matériaux } & \multirow{2}{*}{$\begin{array}{l}\text { Poids pour } 1 \mathrm{~m}^{3} \\
(\mathrm{~kg})\end{array}$} & \multicolumn{2}{|c|}{ Process de fabrication et mise en œuvre } \\
\hline & & $\begin{array}{l}\text { Dégagement de } \\
\mathrm{CO}_{2}(\mathrm{~kg})\end{array}$ & $\begin{array}{l}\text { Fixation de } \mathrm{CO}_{2} \\
(\mathrm{~kg})\end{array}$ \\
\hline Acier . . . . . . . . . . . & 7200 & 5000 & 0 \\
\hline Béton $\ldots \ldots \ldots \ldots \ldots \ldots$ & 2300 & 375 & 0 \\
\hline Production de ciment $\ldots . . .$. & 1600 & 2500 & 0 \\
\hline Bois feuillus $\left(^{*}\right) \ldots \ldots \ldots \ldots \ldots$ & 700 & $\begin{array}{l}\text { Prise en compte des } \\
\text { process industriels } \\
\approx 200\end{array}$ & 1000 \\
\hline
\end{tabular}

(*) si le bois est constitué de $\mathrm{C}=50 \%, 0=43 \%, \mathrm{H}=6 \%, \mathrm{~N}=1 \%$ :

$700 \mathrm{~kg}$ de bois $\Rightarrow 350 \mathrm{~kg}$ de $\mathrm{C}$

1 mole de $\mathrm{C}=12 \mathrm{~g} ; 1$ mole de $\mathrm{O}_{2}=32 \mathrm{~g}$

$\Rightarrow 1$ mole de $\mathrm{CO}_{2}=44 \mathrm{~g}$

$\Rightarrow$ pour $350 \mathrm{~kg}$ de C: $350 / 12 \times 44=1283 \mathrm{~kg}$ de $\mathrm{CO}_{2}$

arrondi à 1 tonne de $\mathrm{CO}_{2}$ par $\mathrm{m}^{3}$ de bois utilisé

Ces chiffres traduisent tout l'intérêt environnemental lié à l'utilisation du bois (donc à la gestion de la forêt). Matériau renouvelable, véritable écomatériau, associé à une gestion forestière bien conduite, il y a, à travers la prise de conscience écologique accompagnée d'une information objective des consommateurs, un réel avenir pour le bois dans les secteurs de la construction.

\section{CONCLUSIONS}

Le bois écomatériau d'avenir, mais aussi matériau enraciné dans l'histoire de nos civilisations s'est fait beaucoup de tort en voulant de manière excessive rester cantonné sur ses valeurs de tradition, de noblesse, de matériau vivant, etc. C'est sans doute en jouant résolument la carte de la modernité et sans renier son passé riche d'enseignements que le bois s'affirmera comme le matériau d'excellence fait pour construire, et ce, en prenant en compte mieux que d'autres l'intégralité des problèmes qui se posent à nos sociétés modernes, en termes d'adéquation avec l'emploi en zones rurales, en termes de qualité de vie dans l'habitat et surtout en termes de respect de l'environnement. C'est bien sur ce dernier point que se situe l'enjeu de notre matériau, et ce rendez-vous, si l'on ne veut pas le manquer, doit associer dans une même démarche forestiers, industriels des produits de la forêt et architectes. Le secteur le plus accessible pour accompagner ce challenge est celui du bois-construction. Au-delà de phénomènes de mode, il s'inscrit dans une tendance à long 
terme parce que la prise de conscience des Maîtres d'œuvre est aujourd'hui une réalité palpable, "les architectes vont trouver dans la construction en bois les moyens d'une expression architecturale différenciée et créative fondée sur la compétence de ceux qui la pensent et de ceux qui la font » (Bignon et al., 2000).

Dans ces périodes d'inquiétudes en l'avenir, il est sans doute normal de revenir à ce qui fut l'un des premiers matériaux de construction de l'Humanité. Ce n'est pas là un retour en arrière, c'est la prise de conscience que le bois n'est, en aucune manière, un matériau du passé mais, en fait, l'un des rares matériaux d'hier, d'aujourd'hui, et de demain, ce qui en fait définitivement le matériau de toujours.
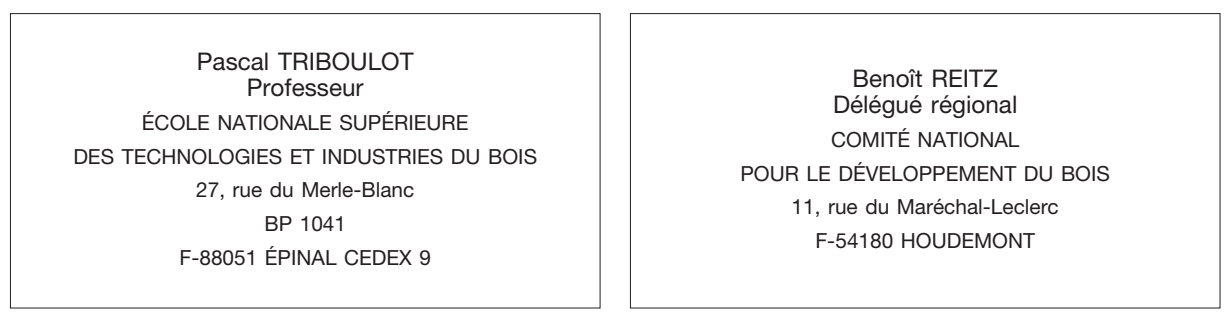

\section{BIBLIOGRAPHIE}

BIGNON (J.-C.), TRIBOULOT (P.), TRIBOULOT (M.-C.), REITZ (B.), BUCHMANN (A.), SANDOZ (J.-L.), DÜBLER (A.). - Bois et Environnement. - Préparation de la conférence nationale CNDB sur les thèmes environnementaux, 1999-2000.

BIANCO (J.-L.). - La Forêt, une chance pour la France. - Rapport parlementaire au Premier Ministre, Ministère de l'Agriculture et de la Pêche, 1998. - Revue forestière française, vol. L, n 6, 1998.

CTBA. - Guide de la préservation du bois. - Paris : CTBA, 1998.

DESBOIS (M.), POURADIER (G.). - La France blessée, autopsie d'une catastrophe climatique. - Éditions Ramsay, mars 2000.

FACOM. - Sélection d'outillages pour les métiers. - Catalogue FACOM, 1994.

GORDON (J.E.). - Structures et matériaux, l'explication mécanique des formes. - Pour la Science Belin Éd., 1994.

GUINARD (D.). - Le Bois écomatériau : un atout pour développer ses emplois. - CTBA Info, n $56,1995$.

HILEMAN (B.). - Case grows for climate change. - Chemical and Engineering News, August 9, 1999.

LEGOVIC (C.). - Les Assemblages dans la construction bois. - Paris : CTBA, 1995.

NATTERER (J.) et al. - Le Choix du bois. - Ingénieurs et Architectes suisses, n² 21, septembre 1995.

NOËL (M.), BOCQUET (A.). - Les Hommes et le bois, histoire et technologie du bois de la préhistoire à nos jours. - Paris : Éditions Hachette, 1987 (La mémoire du temps).

PICON (A.). - L'Art de l'ingénieur, constructeur, entrepreneur, inventeur. - Éditions du Moniteur, 1997 (ISBN : 2858509115).

SANDOZ (J.-L.). - Les Forêts du monde dans l'ombre de Malthus : intérêt ou devoir de construire en bois ? Journal de la Construction, $\mathrm{n}^{\circ}$ 12, 15 décembre 1994.

TRIBOULOT (P.), DUCHANOIS (G.). - Pour une approche novatrice du matériau bois. - Revue technique luxembourgeoise, $\mathrm{n}^{\circ}$ 3, juillet-septembre 1996.

TRIBOULOT (P.), TRIBOULOT (M.-C.). - Bois et matériaux dérivés. - Refonte C925. À paraître $4^{e}$ trimestre 2000 (Techniques de l'Ingénieur - Construction). 


\section{LE BOIS DANS LE CONTEXTE DES MATÉRIAUX DE CONSTRUCTION (Résumé)}

Le bois est, avec la terre, l'un des plus vieux matériaux de construction de l'humanité. Que ce soit en Asie, en Europe, les exemples de technicité et de pérennité de ces structures ne manquent pas. Ces réalisations ont franchi les siècles et montrent aujourd'hui les qualités remarquables du bois dans l'acte de construire. La structure du matériau présente une architecture anatomique très sophistiquée qui en fait un matériau composite naturel d'exception. La plupart des caractéristiques physico-mécaniques résulte de cette anatomie. Matériau combustible, il présente ce paradoxe d'être l'un des matériaux les plus sûrs face au feu lors de sa mise en œuvre dans le bâti. Dans l'approvisionnement mondial en matières premières, le bois occupe une place de choix : celle d'un matériau indéfiniment renouvelable dont le profil écologique le place en tête de tous les matériaux de construction. Le bois se positionne aujourd'hui de manière forte dans l'architecture contemporaine.

\section{TIMBER AS A BUILDING MATERIAL (Abstract)}

Together with earth, wood is one of the most ancient building materials used by mankind. Both in Asia and Europe, examples of technical accomplishments and durability of those structures are plentiful. These achievements have held up through centuries and are proof of the remarkable qualities of wood in the building process. The material's structure offers a highly sophisticated anatomic configuration making it a remarkable natural composite material. Most of its physical and mechanical characteristics derive from this anatomy. In spite of being combustible, wood is surprisingly one of the least hazardous materials used for construction purposes in relation to fire. Timber plays a leading part on the worldwide raw materials market as an indefinitely renewable material whose environmental profile makes it the foremost building material. It is extensively used today in contemporary architecture.

\section{DAS HOLZ IM KANON DER KONSTRUKTIONWERKSTOFFE (Zusammenfassung)}

Holz ist, so wie Erde, einer der ältesten Konstruktionswerkstoffe der Menschheit. Sowohl in Asien als auch Europa gibt es zahlreiche Beispiele für die hohe Technik und Beständigkeit solcher Strukturen. Diese Bauten haben die Jahrhunderte überstanden und zeigen heute die bemerkenswerten Eigenschaften des Holzes in der Konstruktion auf. Die Eigenstruktur des Materials Holz zeigt einen anatomischen, sehr ausgefeilten Aufbau, der es zu einem außergewöhnlichen natürlichen Verbundwerkstoff macht. Die Mehrzahl der physikalisch-mechanischen Eigenschaften resultiert aus diesem Aufbau. Eigentlich ein Brennstoff, ist es paradoxerweise eines der sichersten Materialien gegen Feuer, wenn es im Bau zum Einsatz kommt. Bei der weltweiten Rohstoffversorgung nimmt das Holz einen ausgewählten Platz ein : den eines unendlich erneuerbaren Werkstoffs, dessen Öko-Profil es an die Spitze aller Konstruktionsmaterialien stellt. Das Holz nimmt heute einen wichtigen Platz in der zeitgenössischen Architektur ein.

\section{LA MADERA EN EL CONTEXTO DE LOS MATERIALES DE CONSTRUCCIÓN (Resumen)}

La madera es, con la tierra, uno de los más viejos materiales de construcción de la humanidad. Ya sea en Asia o en Europa, no faltan los ejemplos de tecnicidad y de perennidad de tales estructuras. Esas realizaciones han franqueados los siglos y muestran hoy la cualidades considerables de la madera durante la construcción. La estructura del material presenta una arquitectura anatómica muy sofisticada, constituyendo un material, compuesto natural, de excepción. La mayoría de las caracteristicas fisicos-mecánicas resulta de dicha anatomia. Siendo un material combustible, presenta la paradoja de ser uno de los materiales más seguros frente al fuego, durante su empleo en la armazón. En el aprovisionamiento mundial en materias primas, la madera ocupa un puesto importante : el de un material indefinidamente renovable, cuyo perfil ecológico le coloca a la cabeza de todos los materiales de construcción. La madera se posiciona hoy de manera fuerte en la arquitectura contemporánea. 